\title{
Boundedness for some Schrödinger Type Operators on Weighted Morrey Spaces
}

\author{
Guixia Pan and Lin Tang \\ LMAM, School of Mathematical Sciences, Peking University, Beijing 100871, China \\ Correspondence should be addressed to Lin Tang; tanglin@math.pku.edu.cn \\ Received 3 October 2013; Accepted 18 December 2013; Published 30 January 2014 \\ Academic Editor: Yoshihiro Sawano
}

Copyright ( 2014 G. Pan and L. Tang. This is an open access article distributed under the Creative Commons Attribution License, which permits unrestricted use, distribution, and reproduction in any medium, provided the original work is properly cited.

We establish the boundedness of some Schrödinger type operators on weighted Morrey spaces related to certain nonnegative potentials belonging to the reverse Hölder class.

\section{Introduction}

In this paper, we consider the Schrödinger differential operator

$$
\mathscr{L}=-\Delta+V(x) \text { on } \mathbb{R}^{n}, \quad n \geq 3,
$$

where $V(x)$ is a nonnegative potential belonging to the reverse Hölder class $B_{q}$ for $q \geq n / 2$.

A nonnegative locally $L^{q}$ integrable function $V(x)$ on $\mathbb{R}^{n}$ is said to belong to $B_{q}(q>1)$ if there exists $C>0$ such that the reverse Hölder inequality

$$
\left(\frac{1}{|B|} \int_{B} V^{q} d x\right)^{1 / q} \leq C\left(\frac{1}{|B|} \int_{B} V d x\right)
$$

holds for every ball $B$ in $\mathbb{R}^{n}$; see [1].

For $x \in \mathbb{R}^{n}$, the function $\rho(x)$ is defined by

$$
\rho(x):=\sup _{r>0}\left\{r: \frac{1}{r^{n-2}} \int_{B(x, r)} V(y) d y \leq 1\right\} .
$$

Let $p \in[1, \infty), \alpha \in(-\infty, \infty)$ and $\lambda \in[0,1)$. For $f \in$ $L_{\text {loc }}^{p}\left(\mathbb{R}^{n}\right)$ and $V \in B_{q}(q>1)$, we say $f \in L_{\alpha, V, \omega}^{p, \lambda}\left(\mathbb{R}^{n}\right)$ (weighted Morrey spaces related to the potential $V$ ) provided that

$$
\begin{aligned}
\|f\|_{L_{\alpha, V, \omega}^{p, \lambda}\left(\mathbb{R}^{n}\right)}^{p}= & \sup _{B\left(x_{0}, r\right) \subset \mathbb{R}^{n}}\left(1+\frac{r}{\rho\left(x_{0}\right)}\right)^{\alpha} \omega\left(B\left(x_{0}, 2 r\right)\right)^{-\lambda} \\
& \times \int_{B\left(x_{0}, r\right)}|f(x)|^{p} \omega(x) d x<\infty
\end{aligned}
$$

where $B=B\left(x_{0}, r\right)$ denotes a ball with centered at $x_{0}$ and radius $r$, and the weight functions $\omega \in A_{p}^{\rho, \infty}$ (see Section 2). The space $L_{\alpha, V, \omega}^{p, \lambda}\left(\mathbb{R}^{n}\right)$ could be viewed as an extension of weighted Lebesgue spaces (i.e., when $\alpha=\lambda=0$, $\left.\|f\|_{L_{0, V, \omega}^{p, 0}\left(\mathbb{R}^{n}\right)}=\|f\|_{L_{\omega}^{p}\left(\mathbb{R}^{n}\right)}\right)$. In particular, when $\alpha=0$ or $V=0$, $\omega=1$ and $0<\lambda<1$, the space $L_{\alpha, V, \omega}^{p, \lambda}\left(\mathbb{R}^{n}\right)$ is the classic Morrey space $L^{p, \lambda}\left(\mathbb{R}^{n}\right)$ (see [2]). When $\alpha=0$ or $V=0$ and $0<\lambda<1, L_{\omega}^{p, \lambda}\left(\mathbb{R}^{n}\right)$ was first introduced in [3] (see also [4]), where $\omega \in A_{p}\left(\mathbb{R}^{n}\right)$ (Muckenhoupt weights class). It is easy to see that $L_{\alpha, V, \omega}^{p, \lambda}\left(\mathbb{R}^{n}\right) \subset L_{\omega}^{p, \lambda}\left(\mathbb{R}^{n}\right)$ for $\alpha>0$, and $L_{\omega}^{p, \lambda}\left(\mathbb{R}^{n}\right) \subset L_{\alpha, V, \omega}^{p, \lambda}\left(\mathbb{R}^{n}\right)$ for $\alpha<0$. In addition, when $\omega=1$, the $L_{\alpha, V, \omega}^{p, \lambda}\left(\mathbb{R}^{n}\right)$ has been studied in [5].

From $[1,6]$, we know some Schrödinger type operators, such as $\nabla(-\Delta+V)^{-1} \nabla$ with $V \in B_{n}, \nabla(-\Delta+V)^{-1 / 2}$ with $V \in B_{n},(-\Delta+V)^{-1 / 2} \nabla$ with $V \in B_{n},(-\Delta+V)^{i \gamma}$ with $\gamma \in \mathbb{R}$ and $V \in B_{n / 2}$, and $\nabla^{2}(-\Delta+V)^{-1}$ with $V$ is a nonnegative polynomial, are standard Calderón-Zygmund operators; see [7]. In particular, the kernels $K$ of operators above all satisfy

$$
|K(x, y)| \leq \frac{C_{k}}{(1+(|x-y| / \rho(x)))^{k}} \frac{1}{|x-y|^{n}}
$$

for any $k \in \mathbb{N}$. Hence, in the rest of this paper, we always assume that $T$ denotes the above operators.

Recently, Bongioanni et al. [8] proved $L^{p}\left(\mathbb{R}^{n}\right)(1<$ $p<\infty)$ boundedness for commutators of Riesz transforms associated with Schrödinger operator with $\mathrm{BMO}_{\rho}$ functions 
which include the classic BMO function, and they [9] established the weighted boundedness for Riesz transforms, fractional integrals, and Littlewood-Paley functions associated with Schrödinger operator with weight $A_{p}^{\rho, \infty}$ class which includes the Muckenhoupt weight class. Very recently, the author $[10,11]$ established the weighted norm inequalities for some Schrödinger type operators, which include commutators of Riesz transforms, fractional integrals, and LittlewoodPaley functions with $\mathrm{BMO}_{\rho}$ functions; see also $[12,13]$.

The aim of this paper is to study the boundedness properties of some Schrödinger type operators on the weighted Morrey spaces $\|f\|_{L_{\alpha, V, \omega}^{p, \lambda}\left(\mathbb{R}^{n}\right)}$. Our main results in this paper are formulated as follows.

Theorem 1. Suppose $\alpha \in(-\infty, \infty)$ and $\lambda \in(0,1)$.

(i) If $1<p<\infty$ and $\omega \in A_{p}^{\rho, \infty}$, then

$$
\|T f\|_{L_{\alpha, V, \omega}^{p, \lambda}\left(\mathbb{R}^{n}\right)} \leq C\|f\|_{L_{\alpha, V, \omega}^{p, \lambda}\left(\mathbb{R}^{n}\right)},
$$

where $C$ is independent of $f$.

(ii) If $p=1$ and $\omega \in A_{1}^{\rho, \infty}$, then for any $t>0$,

$$
\begin{aligned}
& \omega(B(x, 2 r))^{-\lambda}\left(1+\frac{r}{\rho(x)}\right)^{\alpha} \\
& \quad \times t \omega(\{y \in B(x, r):|T f(y)|>t\}) \leq C\|f\|_{L_{\alpha, V, \omega}^{1, \lambda}\left(\mathbb{R}^{n}\right)}
\end{aligned}
$$

holds for all balls $B$, where $C$ is independent of $x, r, t$, and $f$.

Let $b \in \mathrm{BMO}_{\rho}$ (see its definition in Section 2); we define the commutator of $T$ by

$$
[b, T] f=b T f-T(b f) .
$$

Theorem 2. Suppose $b \in B M O_{\rho}, \alpha \in(-\infty, \infty)$ and $\lambda \in$ $(0,1)$.

(i) If $1<p<\infty$ and $\omega \in A_{p}^{\rho, \infty}$, then

$$
\|[b, T] f\|_{L_{\alpha, V, \omega}^{p, \lambda}\left(\mathbb{R}^{n}\right)} \leq C\|f\|_{L_{\alpha, V, \omega}^{p, \lambda}\left(\mathbb{R}^{n}\right)}
$$

where $C$ is independent of $f$.

(ii) If $p=1$ and $\omega \in A_{1}^{\rho, \infty}$, then, for any $t>0$,

$$
\begin{aligned}
\omega(B(x, 2 r))^{-\lambda}\left(1+\frac{r}{\rho(x)}\right)^{\alpha} \\
\quad \times \omega(\{y \in B(x, r):|[b, T] f(y)|>t\}) \\
\leq C \sup _{B(x, r) \subset \mathbb{R}^{n}} \omega(B(x, 2 r))^{-\lambda}\left(1+\frac{r}{\rho(x)}\right)^{\alpha} \\
\quad \times \int_{B(x, r)} \frac{|f(y)|}{t} \ln \left(2+\frac{|f(y)|}{t}\right) \omega(y) d y
\end{aligned}
$$

holds for all balls $B(x, r)$, where $C$ is independent of $x$, $r$, $t$, and $f$.
Next, we discuss the Littlewood-Paley $g$ function related to Schrödinger operators defined by

$$
g(f)(x)=\left(\int_{0}^{\infty}\left|\frac{d}{d t} e^{-t L}(f)(x)\right|^{2} t d t\right)^{1 / 2},
$$

and the commutator $g_{b}$ of $g$ with $b \in \operatorname{BMO}(\rho)$ is defined by

$$
g_{b}(f)(x)=\left(\int_{0}^{\infty}\left|\frac{d}{d t} e^{-t L}((b(x)-b(\cdot)) f)(x)\right|^{2} t d t\right)^{1 / 2} \text {. }
$$

Theorem 3. Suppose $V \in B_{n / 2}, \alpha \in(-\infty, \infty)$, and $\lambda \in(0,1)$.

(i) If $1<p<\infty$ and $\omega \in A_{p}^{\rho, \infty}$, then

$$
\|g(f)\|_{L_{\alpha, V, \omega}^{p, \lambda}\left(\mathbb{R}^{n}\right)} \leq C\|f\|_{L_{\alpha, V, \omega}^{p, \lambda}\left(\mathbb{R}^{n}\right)},
$$

where $C$ is independent of $f$.

(ii) If $p=1$ and $\omega \in A_{1}^{\rho, \infty}$, then, for any $t>0$,

$$
\begin{aligned}
& \omega(B(x, 2 r))^{-\lambda}\left(1+\frac{r}{\rho(x)}\right)^{\alpha} \\
& \quad \times t \omega(\{y \in B(x, r):|g(f)(y)|>t\}) \leq C\|f\|_{L_{\alpha, V, \omega}^{1, \lambda}\left(\mathbb{R}^{n}\right)}
\end{aligned}
$$

holds for all balls $B$, where $C$ is independent of $x, r, t$, and $f$.

Theorem 4. Suppose $V \in B_{n / 2}, b \in B M O_{\rho}, \alpha \in(-\infty, \infty)$, and $\lambda \in(0,1)$.

(i) If $1<p<\infty$ and $\omega \in A_{p}^{\rho, \infty}$, then

$$
\left\|g_{b}(f)\right\|_{L_{\alpha, V, \omega}^{p, \lambda}\left(\mathbb{R}^{n}\right)} \leq C\|f\|_{L_{\alpha, V, \omega}^{p, \lambda}\left(\mathbb{R}^{n}\right)},
$$

where $C$ is independent of $f$.

(ii) If $p=1$ and $\omega \in A_{1}^{\rho, \infty}$, then, for any $t>0$,

$$
\begin{aligned}
\omega(B(x, 2 r))^{-\lambda}\left(1+\frac{r}{\rho(x)}\right)^{\alpha} \\
\times \omega\left(\left\{y \in B(x, r):\left|g_{b}(f)(y)\right|>t\right\}\right) \\
\leq C \sup _{B(x, r) \subset \mathbb{R}^{n}}\left(1+\frac{r}{\rho(x)}\right)^{\alpha} \omega(B(x, 2 r))^{-\lambda} \\
\quad \times \int_{B(x, r)} \frac{|f(y)|}{t} \ln \left(2+\frac{|f(y)|}{t}\right) \omega(y) d y
\end{aligned}
$$

holds for all balls $B$, where $C$ is independent of $x, r, t$, and $f$.

Finally, we consider the boundedness of fractional integrals related to Schrödinger operators.

Let $\mathscr{L}=-\Delta+V$ with $V \in B_{q}$ for $q \geq n / 2$ and its associated semigroup:

$$
\begin{array}{r}
T_{t} f(x)=e^{-t \mathscr{L}} f(x)=\int_{\mathbb{R}^{n}} k_{t}(x, y) f(y) d y, \\
f \in L^{2}\left(\mathbb{R}^{n}\right), \quad t>0 .
\end{array}
$$


The $\mathscr{L}$-fractional integral operator is defined by

$$
\begin{aligned}
\mathscr{I}_{\beta} f(x) & =\mathscr{L}^{-\beta / 2} f(x) \\
& =\int_{0}^{\infty} e^{-t \mathscr{L}} f(x) t^{\beta / 2-1} d t \quad \text { for } 0<\beta<n .
\end{aligned}
$$

Theorem 5. Suppose $V \in B_{n / 2}, \alpha \in(-\infty, \infty)$ and $0<\beta<n$.

(i) If $1<p<n / \beta, 1 / q=1 / p-\beta / n, v=q / p, 0<\lambda<$ $1 / \nu$, and $\omega^{q} \in A_{1+q / p^{\prime}}^{\rho, \infty}$, where $p^{\prime}=p /(p-1)$, then

$$
\left\|\mathscr{I}_{\beta} f\right\|_{L_{\alpha, V, \omega}^{q, \nu \lambda}\left(\mathbb{R}^{n}\right)} \leq C\|f\|_{L_{\alpha, V, \omega}^{p, \lambda} p}\left(\mathbb{R}^{n}\right)^{p}
$$

where $C$ is independent of $f$.

(ii) If $p=1, q=n /(n-\beta), 0<\lambda<1$, and $\omega \in A_{1}^{\rho, \infty}$, then, for any $t>0$,

$$
\begin{aligned}
& \omega(B(x, 2 r))^{-\lambda}\left(1+\frac{r}{\rho(x)}\right)^{\alpha} \\
& \quad \times t \omega\left(\left\{y \in B(x, r):\left|\mathscr{I}_{\beta} f(y)\right|>t\right\}\right)^{1 / q} \leq C\|f\|_{L_{\alpha, V, \omega}^{1, \lambda}, q}\left(\mathbb{R}^{n}\right)
\end{aligned}
$$

holds for all balls $B(x, r)$, where $C$ is independent of $x$, $r, t$, and $f$.

Let $b \in \mathrm{BMO}_{\rho}$; we define the commutator of $\mathscr{I}_{\beta}$ by

$$
\left[b, \mathscr{I}_{\beta}\right] f=b \mathscr{I}_{\beta} f-\mathscr{I}_{\beta}(b f) .
$$

Theorem 6. Let $b \in B M O, V \in B_{n / 2}, \alpha \in(-\infty, \infty)$, and $0<\beta<n$.

(i) If $1<p<n / \beta, 1 / q=1 / p-\beta / n, v=q / p, 0<\lambda<$ $1 / \nu$, and $\omega^{q} \in A_{1+q / p^{\prime}}^{\rho, \infty}$, then

$$
\left\|\left[b, \mathscr{I}_{\beta}\right] f\right\|_{L_{\alpha, V, \omega}^{q, v \lambda}\left(\mathbb{R}^{n}\right)} \leq C\|f\|_{L_{\alpha, V, \omega}^{p, \lambda}\left(\mathbb{R}^{n}\right)}
$$

where $C$ is independent of $f$.

(ii) If $p=1, q=n /(n-\beta), 0<\lambda<1$, and $\omega \in A_{1}^{\rho, \infty}$, then, for any $t>0$,

$$
\begin{aligned}
\omega(B(x, 2 r))^{-\lambda}\left(1+\frac{r}{\rho(x)}\right)^{\alpha} \\
\quad \times \omega\left(\left\{y \in B(x, r):\left|\left[b, \mathscr{I}_{\beta}\right] f(y)\right|>t\right\}\right) \\
\leq C \sup _{B(x, r) \subset \mathbb{R}^{n}} \omega(B(x, 2 r))^{-\lambda}\left(1+\frac{r}{\rho(x)}\right)^{\alpha} \\
\quad \times \Phi\left(\int_{B} \frac{|f(y)|}{t} \ln \left(2+\frac{|f(y)|}{t}\right) \Theta(\omega(y)) d y\right)
\end{aligned}
$$

holds for all balls $B=B(x, r)$, where $\Phi(t)=[t \log (2+$ $\left.\left.t^{\beta / n}\right)\right]^{n /(n-\beta)}$ and $\Theta(t)=t^{1-\beta / n} \log \left(e+t^{-\beta / n}\right)$, and $C$ is independent of $x, r, t$, and $f$.
We remark that even if in the $\omega=1$ case, Theorems 2, 3, 4 , and 6 are also new; see [5].

Throughout this paper, $C$ is a positive constant which is independent of the main parameters and not necessary the same at each occurrence.

\section{Some Notation and Basic Results}

We first recall some notation. Given $B=B(x, r)$ and $\lambda>0$, we will write $\lambda B$ for the $\lambda$-dilate ball, which is the ball with the same center $x$ and with radius $\lambda r$. Given a Lebesgue measurable set $E$ and a weight $\omega,|E|$ will denote the Lebesgue measure of $E$ and $\omega(E)=\int_{E} \omega d x .\|f\|_{L^{p}(\omega)}$ will denote $\left(\int_{\mathbb{R}^{n}}|f(y)|^{p} \omega(y) d y\right)^{1 / p}$ for $0<p<\infty$.

Lemma 7 (see [1]). There exists $l_{0}>0$ and $C_{0}>1$ such that

$$
\frac{1}{C_{0}}\left(1+\frac{|x-y|}{\rho(x)}\right)^{-l_{0}} \leq \frac{\rho(y)}{\rho(x)} \leq C_{0}\left(1+\frac{|x-y|}{\rho(x)}\right)^{l_{0} /\left(l_{0}+1\right)} .
$$

In particular, $\rho(x) \sim \rho(y)$ if $|x-y|<C \rho(x)$.

In this paper, we write $\Psi_{\theta}(B)=\left(1+r / \rho\left(x_{0}\right)\right)^{\theta}$, where $\theta>$ $0, x_{0}$ and $r$ denotes the center and radius of $B$, respectively.

A weight will always mean a nonnegative function which is locally integrable. As in [9], we say that a weight $\omega$ belongs to the class $A_{p}^{\rho, \theta}$ for $1<p<\infty$, if there is a constant $C$ such that for all ball $B=B(x, r)$

$$
\begin{aligned}
& \left(\frac{1}{\Psi_{\theta}(B)|B|} \int_{B} \omega(y) d y\right) \\
& \quad \times\left(\frac{1}{\Psi_{\theta}(B)|B|} \int_{B} \omega^{-1 /(p-1)}(y) d y\right)^{p-1} \leq C .
\end{aligned}
$$

We also say that a nonnegative function $\omega$ satisfies the $A_{1}^{\rho, \theta}$ condition if there exists a constant $C$ for all balls $B$

$$
M_{V}^{\theta}(\omega)(x) \leq C \omega(x), \quad \text { a.e. } x \in \mathbb{R}^{n}
$$

where

$$
M_{V}^{\theta} f(x)=\sup _{x \in B} \frac{1}{\Psi_{\theta}(B)|B|} \int_{B}|f(y)| d y .
$$

Since $\Psi_{\theta}(B) \geq 1$, obviously, $A_{p} \subset A_{p}^{\rho, \theta}$ for $1 \leq p<\infty$, where $A_{p}$ denote the classical Muckenhoupt weights; see [7]. We will see that $A_{p} \subset A_{p}^{\rho, \theta}$ for $1 \leq p<\infty$ in some cases. In fact, let $\theta>0$ and $0 \leq \gamma \leq \theta$; it is easy to check that $\omega(x)=(1+|x|)^{-(n+\gamma)} \notin A_{\infty}=\bigcup_{p \geq 1} A_{p}$ and $\omega(x) d x$ is not a doubling measure, but $\omega(x)=(1+|x|)^{-(n+\gamma)} \in A_{1}^{\rho, \theta}$ provided that $V=1$ and $\Psi_{\theta}\left(B\left(x_{0}, r\right)\right)=(1+r)^{\theta}$.

For convenience, we always assume that $\Psi(B)$ denotes $\Psi_{\theta}(B), A_{p}^{\rho, \infty}=\bigcup_{\theta>0} A_{p}^{\rho, \theta}$, and $A_{\infty}^{\rho, \infty}=\bigcup_{p \geq 1} A_{p}^{\rho, \infty}$.

Lemma 8 (see [10]). Let $0<\theta<\infty$; then

(i) if $1 \leq p_{1}<p_{2}<\infty$, then $A_{p_{1}}^{\rho, \theta} \subset A_{p_{2}}^{\rho, \theta}$. 
(ii) $\omega \in A_{p}^{\rho, \theta}$ if and only if $\omega^{-1 /(p-1)} \in A_{p^{\prime}}^{\rho, \theta}$, where $1 / p+$ $1 / p^{\prime}=1$.

(iii) If $\omega \in A_{p}^{\rho, \theta}$ for $1 \leq p<\infty$, then there exists a constant such that for any $\lambda>1$

$$
\omega\left(\lambda B\left(x_{0}, r\right)\right) \leq C\left(1+\frac{\lambda r}{\rho\left(x_{0}\right)}\right)^{\left(l_{0}+1\right) \theta} \omega\left(B\left(x_{0}, r\right)\right) .
$$

Lemma 9 (see [12]). Let $0<\theta<\infty, 1 \leq p<\infty$. If $\omega \in A_{p}^{\rho, \theta}$, then there exists positive constants $\delta, \eta$, and $C$ such that

$$
\begin{aligned}
& \left(\frac{1}{|B|} \int_{B} \omega(y)^{1+\delta} d y\right)^{1 /(1+\delta)} \\
& \quad \leq C \frac{1}{|B|} \int_{B} \omega(y) d y\left(1+\frac{r}{\rho\left(x_{0}\right)}\right)^{\eta}
\end{aligned}
$$

for all ball $B\left(x_{0}, r\right)$. result.

As a consequence of Lemma 9, we have the following

Corollary 10 (see [12]). Let $0<\theta<\infty, 1 \leq p<\infty$. If $\omega \in A_{p}^{\rho, \theta}$, then there exist positive constants $q>1, \eta$ and $C$ such that

$$
\frac{\omega(E)}{\omega(B)} \leq C\left(\frac{|E|}{|B|}\right)^{1 / q}\left(1+\frac{r}{\rho\left(x_{0}\right)}\right)^{\eta}
$$

for any measurable subset $E$ of a ball $B\left(x_{0}, r\right)$.

Bongioanni et al. [8] introduce a new space $\mathrm{BMO}_{\theta}(\rho)$ defined by

$$
\|f\|_{\mathrm{BMO}_{\theta}(\rho)}=\sup _{B \subset \mathbb{R}^{n}} \frac{1}{\Psi_{\theta}(B)|B|} \int_{B}\left|f(x)-f_{B}\right| d x<\infty,
$$

where $f_{B}=(1 /|B|) \int_{B} f(y) d y$ and $\Psi_{\theta}(B)=\left(1+r / \rho\left(x_{0}\right)\right)^{\theta}$ and $\theta>0$.

In particular, Bongioanni et al. [8] proved the following result for $\mathrm{BMO}_{\theta}(\rho)$.

Proposition 11. Let $\theta>0$ and $1 \leq s<\infty$. If $b \in B M O_{\theta}(\rho)$, then

$$
\left(\frac{1}{|B|} \int_{B}\left|b-b_{B}\right|^{s}\right)^{1 / s} \leq c C_{0}^{\theta} s\|b\|_{B M O_{\theta}(\rho)}\left(1+\frac{r}{\rho\left(x_{0}\right)}\right)^{\theta^{\prime}},
$$

for all $B=B\left(x_{0}, r\right)$, with $x \in \mathbb{R}^{n}$ and $r>0$, where $\theta^{\prime}=\left(l_{0}+1\right) \theta$ and $C_{0}$ is defined in Lemma 7 and $c$ is a constant depending only on $n$.

Obviously, the classical $\mathrm{BMO}$ is properly contained in $\mathrm{BMO}_{\theta}(\rho)$; for more examples, see [8]. For convenience, we let $\mathrm{BMO} \rho=\bigcup_{\theta>0} \operatorname{BMO}_{\theta}(\rho)$.

Tang [10] gave the following result, which is equivalent to Proposition 11.
Proposition 12. Suppose that $f \in B^{\prime} O_{\theta}(\rho)$. There exist positive constants $\gamma$ and $C$ such that for any ball $B=B\left(x_{0}, r\right)$

$$
\frac{1}{|B|} \int_{B} \exp \left\{\frac{\gamma}{\|f\|_{B M O_{\theta}(\rho)} \Psi_{\theta^{\prime}}(B)}\left|f(x)-f_{B}\right|\right\} d x \leq C .
$$

Applying Corollary 10 and Proposition 11, we can obtain the following result.

Proposition 13. If $f \in \mathrm{BMO}_{\theta}(\rho)$ and $\omega \in A_{p}^{\rho, \theta}(p>1)$, then there exist positive constants $c_{1}, c_{2}$, and $\eta$ such that for every ball $B=B(x, r)$ and every $\lambda>0$, one has

$$
\begin{aligned}
\omega(\{x & \left.\left.\in B:\left|f(x)-f_{B}\right|>\lambda\right\}\right) \\
& \leq c_{1} \omega(B) \exp \left\{-\frac{c_{2} \lambda}{\|f\|_{B M O_{\theta}(\rho)} \Psi_{\theta^{\prime}}(B)}\right\}\left(1+\frac{r}{\rho(x)}\right)^{\eta},
\end{aligned}
$$

where $f_{B}=(1 /|B|) \int_{B} f(y) d y, \Psi_{\theta^{\prime}}(B)=\left(1+r / \rho\left(x_{0}\right)\right)^{\theta^{\prime}}$ and $\theta^{\prime}=\left(l_{0}+1\right) \theta$.

Proof. We adapt the same argument of pages 145-146 in [7]. We first assume $\|f\|_{\mathrm{BMO}(\rho)} \Psi_{\theta^{\prime}}(B)=1$. We apply Chebysheff's inequality and Proposition 11; we obtain

$$
\left|\left\{x \in B:\left|f(x)-f_{B}\right|>\lambda\right\}\right| \leq\left(c C_{0}^{\theta} s\right)^{s} \lambda^{-s}|B|
$$

for $0<\lambda<\infty, 1 \leq s<\infty$. From this and by Corollary 10, there exist constants $q>1$ and $\eta$ such that

$$
\begin{aligned}
\omega(\{x & \left.\left.\in B:\left|f(x)-f_{B}\right|>\lambda\right\}\right) \\
& \leq\left(c C_{0}^{\theta} s\right)^{s / q} \lambda^{-s / q} \omega(B)\left(1+\frac{r}{\rho(x)}\right)^{\eta},
\end{aligned}
$$

for $0<\lambda<\infty, 1 \leq s<\infty$.

If $\lambda \geq 2 c C_{0}^{\theta} / q$, we take $s=\lambda /\left(2 c C_{0}^{\theta} / q\right) \geq 1$. Then

$$
\begin{aligned}
\omega\left(\left\{x \in B:\left|f(x)-f_{B}\right|>\lambda\right\}\right) & \leq\left(\frac{1}{2}\right)^{s} \omega(B)\left(1+\frac{r}{\rho(x)}\right)^{\eta} \\
& =e^{-c_{1} \lambda} \omega(B)\left(1+\frac{r}{\rho(x)}\right)^{\eta}
\end{aligned}
$$

where $c_{1}=\left(2 c C_{0}^{\theta} / q\right)^{-1} \ln 2$. However, if $\lambda \leq 2 c C_{0}^{\theta} / q$, then $e^{-c_{1} \lambda} \geq e^{-c_{1} 2\left(c C_{0}^{\theta} / q\right)}=1 / 2$, and

$$
\omega\left(\left\{x \in B:\left|f(x)-f_{B}\right|>\lambda\right\}\right) \leq 2 e^{-c_{1} \lambda} \omega(B)\left(1+\frac{r}{\rho(x)}\right)^{\eta}
$$

in that range of $\lambda$. Altogether then, if we drop the normalization on $f$ by replacing $f$ by $f /\left(\|f\|_{\mathrm{BMO}_{\theta}(\rho)} \Psi_{\theta^{\prime}}(B)\right)$, we can obtain the conclusion by taking $c_{1}=2$ and $c_{2}=$ $\left(2 c C_{0}^{\theta} / q\right)^{-1} \ln 2$.

From Proposition 13, it is easy to see the following. 
Corollary 14. If $f \in B M O_{\rho}$ and $\omega \in A_{\infty}^{\rho, \infty}$, then there exist positive constants $C$ and $\eta$ such that for every ball $B=B(x, r)$, one has

$$
\frac{1}{\omega(B)} \int_{B}\left|f(x)-f_{B}\right| \omega(x) d x \leq\left(1+\frac{r}{\rho(x)}\right)^{\eta}\|f\|_{B M O_{\rho}}^{p},
$$

where $f_{B}=(1 /|B|) \int_{B} f(y) d y$.

\section{Proof of Theorems 1-4}

Proof of Theorem 1. Without loss of generality, we may assume that $\alpha<0$ and $\omega \in A_{p}^{\rho, \theta}$. Pick any ball $B=B\left(x_{0}, r\right)$ and write

$$
f(x)=f_{0}(x)+\sum_{i=1}^{\infty} f_{i}(x)
$$

where $f_{0}=\chi_{B\left(x_{0}, 2 r\right)} f, f_{i}=\chi_{B\left(x_{0}, 2^{i+1} r\right) \backslash B\left(x_{0}, 2^{i} r\right)} f$ for $i \geq 1$. Hence, we have

$$
\begin{gathered}
\left(\int_{B\left(x_{0}, r\right)}|T f(x)|^{p} \omega(x) d x\right)^{1 / p} \\
\leq\left(\int_{B\left(x_{0}, r\right)}\left|T f_{0}(x)\right|^{p} d x\right)^{1 / p} \\
\quad+\sum_{i=1}^{\infty}\left(\int_{B\left(x_{0}, r\right)}\left|T f_{i}(x)\right|^{p} \omega(x) d x\right)^{1 / p} .
\end{gathered}
$$

By the $L_{\omega}^{p}$ boundedness of $T$ (see [10]), we obtain

$$
\begin{aligned}
\int_{B\left(x_{0}, r\right)}\left|T f_{0}(x)\right|^{p} \omega(x) d x \leq & C\left(1+\frac{r}{\rho\left(x_{0}\right)}\right)^{-\alpha} \\
& \times \omega\left(B\left(x_{0}, 2 r\right)\right)^{\lambda}\|f\|_{L_{\alpha, V, \omega}^{p, \lambda}\left(\mathbb{R}^{n}\right)}^{p}
\end{aligned}
$$

By Lemma 8 and Corollary 10, as well as (5), there exist some positive constants $q>1$ and $\eta$ such that

$$
\begin{aligned}
\int_{B\left(x_{0}, r\right)}\left|T f_{i}(x)\right|^{p} \omega(x) d x & \\
\leq & C \int_{B\left(x_{0}, r\right)}\left(\int_{B\left(x_{0}, 2^{i+1} r\right) \backslash B\left(x_{0}, 2^{i} r\right)}|K(x, y) f(y)| d y\right)^{p} \\
& \times \omega(x) d x \\
\leq & C_{k} \omega\left(B\left(x_{0}, 2^{i+1} r\right)\right)^{\lambda-1}\|f\|_{L_{\alpha, V, \omega}^{p, \lambda}\left(\mathbb{R}^{n}\right)}^{p} \\
& \times \int_{B\left(x_{0}, r\right)} \frac{\left(1+\left(2^{i} r / p\left(x_{0}\right)\right)\right)^{-\alpha+\left(l_{0}+n+4\right) p \theta}}{\left(1+\left(2^{i} r / \rho(x)\right)\right)^{k p}} d x
\end{aligned}
$$

$$
\begin{aligned}
& \leq C_{k} \omega\left(B\left(x_{0}, 2^{i+1} r\right)\right)^{\lambda-1} \omega\left(B\left(x_{0}, r\right)\right) \\
& \times \frac{\left(1+\left(2^{i} r / p\left(x_{0}\right)\right)\right)^{-\alpha+\left(l_{0}+n+4\right) p \theta}}{\left(1+\left(2^{i} r / \rho\left(x_{0}\right)\right)\right)^{k p /\left(l_{0}+1\right)}} \\
& \quad \times\|f\|_{L_{\alpha, V, \omega}^{p, \lambda}}^{p}\left(\mathbb{R}^{n}\right) \\
& \leq C_{k}\left(\frac{\omega\left(B\left(x_{0}, 2 r\right)\right)}{\omega\left(B\left(x_{0}, 2^{i+1} r\right)\right)}\right)^{1-\lambda} \omega\left(B\left(x_{0}, 2 r\right)\right)^{\lambda} \\
& \quad \times \frac{\left(1+\left(2^{i} r / p\left(x_{0}\right)\right)\right)^{-\alpha+\left(l_{0}+n+4\right) p \theta}}{\left(1+\left(2^{i} r / \rho\left(x_{0}\right)\right)\right)^{k p /\left(l_{0}+1\right)}} \\
& \quad \times\|f\|_{L_{\alpha, V}^{p, \lambda}\left(\mathbb{R}^{n}\right)}^{p}
\end{aligned}
$$$$
\leq C_{k} 2^{-i n(1-\lambda) / q} \omega\left(B\left(x_{0}, 2 r\right)\right)^{\lambda}
$$$$
\times \frac{\left(1+\left(2^{i} r / p\left(x_{0}\right)\right)\right)^{-\alpha+\left(l_{0}+n+4\right) p \theta+\eta}}{\left(1+\left(2^{i} r / \rho\left(x_{0}\right)\right)\right)^{k p /\left(l_{0}+1\right)}}
$$$$
\times\|f\|_{L_{\alpha, V}^{p, \lambda}\left(\mathbb{R}^{n}\right)}^{p} \cdot
$$

From (41), (42), and (43) with $k=2\left(\left[-\alpha+\left(l_{0}+n+4\right) p \theta+\right.\right.$ $\eta]+1)\left(l_{0}+1\right)$, we obtain

$$
\|T f\|_{L_{\alpha, V, \omega}^{p, \lambda}\left(\mathbb{R}^{n}\right)} \leq C\|f\|_{L_{\alpha, V, \omega}^{p, \lambda}\left(\mathbb{R}^{n}\right)} .
$$

As for the case $p=1$, the proof can be given by replacing (42) with the corresponding weak estimate.

Proof of Theorem 2. Without loss of generality, we may assume that $\alpha<0, b \in \mathrm{BMO}_{\theta}(\rho)$. Pick any ball $B=B\left(x_{0}, r\right)$, as in the proof of Theorem 1 we write

$$
f(x)=f_{0}(x)+\sum_{i=1}^{\infty} f_{i}(x)
$$

Set $\omega \in A_{p}^{\rho, \infty}$ for $p>1$. By the $L_{\omega}^{p}$ boundedness of $[b, T]$ (see $[10])$, we get

$$
\begin{aligned}
& \int_{B\left(x_{0}, r\right)}\left|[b, T] f_{0}(x)\right|^{p} \omega(x) d x \\
& \quad \leq C\left(1+\frac{r}{\rho(x)}\right)^{-\alpha} \omega\left(B\left(x_{0}, 2 r\right)\right)^{\lambda}\|f\|_{L_{\alpha, V, \omega}^{p, \lambda}\left(\mathbb{R}^{n}\right)}^{p} .
\end{aligned}
$$

Set

$$
b_{r}=\frac{1}{\left|B\left(x_{0}, r\right)\right|} \int_{B\left(x_{0}, r\right)} b(x) d x
$$


When $i \geq 1$, by Lemmas 7 and 9 and Corollaries 10 and 14, there exist some positive constants $q>1$ and $\eta$ such that

$$
\begin{aligned}
& \int_{B\left(x_{0}, r\right)}\left|[b, T] f_{i}(x)\right|^{p} \omega(x) d x \\
& \leq \frac{C_{k}}{\left(1+\left(2^{i} r / \rho\left(x_{0}\right)\right)\right)^{k p /\left(l_{0}+1\right)}} 2^{-i n p} \\
& \times\left\{\int_{B\left(x_{0}, r\right)}\left|b(x)-b_{r}\right|^{p} \omega(x) d x \int_{B\left(x_{0}, 2^{i+1} r\right)}|f(y)| d y\right. \\
& \left.+\omega\left(B\left(x_{0}, r\right)\right) \int_{B\left(x_{0}, 2^{i+1} r\right)}\left|b(y)-b_{r}\right||f(y)| d y\right\} \\
& \leq C_{k} i^{p} \omega\left(B\left(x_{0}, 2^{i} r\right)\right)^{1-\lambda} \omega\left(B\left(x_{0}, 2 r\right)\right)^{\lambda} \\
& \times \frac{\left(1+\left(2^{i} r / \rho\left(x_{0}\right)\right)\right)^{-\alpha+\left(l_{0}+n+4\right) p \theta}}{\left(1+\left(2^{i} r / \rho\left(x_{0}\right)\right)\right)^{k p /\left(l_{0}+1\right)}}\|f\|_{L_{\alpha, V, \omega}^{p, \lambda}\left(\mathbb{R}^{n}\right)}^{p} \\
& \leq C_{k} i\left(\frac{\omega\left(B\left(x_{0}, 2 r\right)\right)}{\omega\left(B\left(x_{0}, 2^{i+1} r\right)\right)}\right)^{1-\lambda} \omega\left(B\left(x_{0}, 2 r\right)\right)^{\lambda} \\
& \times \frac{\left(1+\left(2^{i} r / \rho\left(x_{0}\right)\right)\right)^{-\alpha+\left(l_{0}+n+4\right) p \theta+\eta}}{\left(1+\left(2^{i} r / \rho\left(x_{0}\right)\right)\right)^{k p /\left(l_{0}+1\right)}}\|f\|_{L_{\alpha, V, \omega}^{p, \lambda}}^{p}\left(\mathbb{R}^{n}\right) \\
& \leq C_{k} i 2^{-i n(1-\lambda) / q} \omega\left(B\left(x_{0}, 2 r\right)\right)^{\lambda} \\
& \times \frac{\left(1+\left(2^{i} r / \rho\left(x_{0}\right)\right)\right)^{-\alpha+\left(l_{0}+n+4\right) p \theta+\eta}}{\left(1+\left(2^{i} r / \rho\left(x_{0}\right)\right)\right)^{k p /\left(l_{0}+1\right)}}\|f\|_{L_{\alpha, V, \omega}^{p, \lambda}\left(\mathbb{R}^{n}\right)}^{p} .
\end{aligned}
$$

If we take $k=2\left(\left[-\alpha+\left(l_{0}+n+4\right) p \theta+\eta\right]+1\right)\left(l_{0}+1\right)$ in $(48)$, then we obtain

$$
\|[b, T] f\|_{L_{\alpha, V, \omega}^{p, \lambda}\left(\mathbb{R}^{n}\right)} \leq C\|f\|_{L_{\alpha, V, \omega}^{p, \lambda}\left(\mathbb{R}^{n}\right)} .
$$

It remains to consider the case $p=1$. Set $\omega \in A_{1}^{\rho, \theta}$. From [10], we know that for any $t>0$

$$
\begin{aligned}
\omega(\{y & \left.\left.\in \mathbb{R}^{n}:|[b, T] f(y)|>t\right\}\right) \\
& \leq C \int_{\mathbb{R}^{n}} \frac{|f(x)|}{t} \ln \left(2+\frac{|f(x)|}{t}\right) \omega(x) d x .
\end{aligned}
$$

From this, we have

$$
\begin{aligned}
& \omega\left(B\left(x_{0}, 2 r\right)\right)^{-\lambda}\left(1+\frac{r}{\rho\left(x_{0}\right)}\right)^{\alpha} \\
& \times \omega\left(\left\{y \in B\left(x_{0}, r\right):\left|[b, T] f_{0}(y)\right|>t\right\}\right) \\
& \leq C\left(1+\frac{r}{\rho\left(x_{0}\right)}\right)^{\alpha} \omega\left(B\left(x_{0}, 2 r\right)\right) \\
& \times \int_{B\left(x_{0}, 2 r\right)} \frac{|f(x)|}{t} \ln \left(2+\frac{|f(x)|}{t}\right) \omega(x) d x
\end{aligned}
$$

Set

$$
b_{2^{i+1} r}=\frac{1}{\left|B\left(x_{0}, 2^{i+1} r\right)\right|} \int_{B\left(x_{0}, 2^{i+1} r\right)} b(x) d x .
$$

When $i \geq 1$, by Lemma 7, Corollary 10, and Proposition 12, note that $\omega \in A_{1}^{\rho, \theta}$, then there exist some positive constants $q>1$ and $\eta$ such that

$$
\begin{aligned}
& \omega\left(B\left(x_{0}, 2 r\right)\right)^{-\lambda} t^{-1}\left(1+\frac{r}{\rho\left(x_{0}\right)}\right)^{\alpha} \\
& \times \int_{B\left(x_{0}, r\right)}\left|[b, T] f_{i}(y) \omega(y)\right| d y \\
& \leq \frac{C_{k}\left(1+\left(2^{i} r / \rho\left(x_{0}\right)\right)\right)^{\alpha+\left(l_{0}+n+4\right) p \theta+\eta}}{\left(1+\left(r / \rho\left(x_{0}\right)\right)\right)^{k /\left(l_{0}+1\right)}} \\
& \times\left(2^{i} r\right)^{-n} \omega\left(B\left(x_{0}, 2 r\right)\right)^{-\lambda} t^{-1}\left(1+\frac{r}{\rho\left(x_{0}\right)}\right)^{\alpha} \\
& \times\left\{\int_{B\left(x_{0}, r\right)}\left|b(y)-b_{2^{i+1} r}\right| \omega(x) d x \int_{B\left(x_{0}, 2^{i+1} r\right)}|f(y)| d y\right. \\
& \left.+\omega\left(B\left(x_{0}, r\right)\right) \int_{B\left(x_{0}, 2^{i+1} r\right)}\left|b(y)-b_{2^{i+1} r}\right||f(y)| d y\right\} \\
& \leq C \omega\left(B\left(x_{0}, 2 r\right)\right)^{-\lambda} \omega(B) t^{-1}\left(1+\frac{r}{\rho\left(x_{0}\right)}\right)^{-3 k_{0}} \\
& \times\left(\left\|b-b_{2^{i+1} r}\right\|_{\exp L, B_{i}}\|f\|_{L \log L, B_{i}}+i \omega\left(B_{i}\right)^{-1}\right. \\
& \left.\times\|b\|_{\mathrm{BMO}_{\theta}(\rho)} \int_{B_{i}}|f(y)| \omega(y) d y\right) \\
& \leq \operatorname{Ci\omega }\left(B\left(x_{0}, 2 r\right)\right)^{-\lambda} \omega(B) t^{-1}\left(1+\frac{r}{\rho\left(x_{0}\right)}\right)^{-3 k_{0}} \\
& \times \inf _{\gamma>0}\left\{\gamma+\frac{\gamma}{\left|B_{i}\right|} \int_{B_{i}} \frac{|f(y)|}{\gamma} \log \left(2+\frac{|f(y)|}{\gamma}\right) d y\right\} \\
& +\operatorname{Ci\omega }\left(B\left(x_{0}, 2 r\right)\right)^{-\lambda} \omega(B) \omega\left(B_{i}\right)^{-1} t^{-1}\left(1+\frac{r}{\rho\left(x_{0}\right)}\right)^{-3 k_{0}} \\
& \times \int_{B_{i}}|f(y)| \omega(y) d y \\
& \leq C i\left(\frac{\omega(2 B)}{\omega\left(B_{i}\right)}\right)^{1-\lambda} \omega\left(2 B_{i}\right)^{-\lambda}\left(1+\frac{r}{\rho\left(x_{0}\right)}\right)^{-k_{0}} \\
& \times \int_{B_{i}} \frac{|f(y)|}{t} \log \left(2+\frac{|f(y)|}{t}\right) \omega(y) d y \\
& \leq C i 2^{-i n(1-\lambda) / q} \omega\left(2 B_{i}\right)^{-\lambda}\left(1+\frac{r}{\rho\left(x_{0}\right)}\right)^{\alpha} \\
& \times \int_{B_{i}} \frac{|f(y)|}{t} \log \left(2+\frac{|f(y)|}{t}\right) \omega(y) d y,
\end{aligned}
$$


by taking $k=\left(2\left[-\alpha+\left(l_{0}+n+4\right) p \theta+\eta\right]+1\right)\left(l_{0}+1\right)$ and $k=4 k_{0}$, where in the sixth inequality, we used the following facts (see [2]);

$$
\begin{gathered}
\|f\|_{L \log L, B, \omega}=\inf \left\{\gamma>0: \frac{1}{|B|} \int_{B} \frac{|f(y)|}{\gamma}\right. \\
\left.\times \log \left(2+\frac{|f(y)|}{\gamma}\right) d y \leq 10\right\}, \\
\|f\|_{\exp L, B}=\inf \left\{\gamma>0: \frac{1}{|B|} \int_{B} \exp \left(\frac{|f(y)|}{\gamma}\right) d y \leq 10\right\} ;
\end{gathered}
$$

the generalized Hölder inequality

$$
\begin{gathered}
\frac{1}{|B|} \int_{B}|f(y) h(y)| d y \leq C\|f\|_{L \log L, B}\|h\|_{\exp L, B}, \\
\|f\|_{L \log L, B} \leq \inf _{\lambda>0}\left\{\gamma+\frac{\gamma}{|B|} \int_{B} \frac{|f(y)|}{\gamma} \log \left(2+\frac{|f(y)|}{\gamma}\right) d y\right\} \\
\leq 2\|f\|_{L \log L, B} .
\end{gathered}
$$

Combing (51) and (53), we obtain that

$$
\begin{aligned}
\omega\left(B\left(x_{0}, 2 r\right)\right)^{-\lambda}\left(1+\frac{r}{\rho\left(x_{0}\right)}\right)^{\alpha} \\
\quad \times \omega(\{y \in B(x, r):|[b, T] f(y)|>t\}) \\
\leq C \sup _{B(x, r) \subset \mathbb{R}^{n}}\left(1+\frac{r}{\rho\left(x_{0}\right)}\right)^{\alpha} \omega\left(B\left(x_{0}, 2 r\right)\right)^{-\lambda} \\
\quad \times \int_{B(x, r)} \frac{|f(y)|}{t} \ln \left(2+\frac{|f(y)|}{t}\right) \omega(y) d y
\end{aligned}
$$

holds for all balls $B$, where $C$ is independent of $x, r, t$, and $f$.

Thus, Theorem 2 is proved.

Finally, we give some sketch proof of Theorems 3 and 4 .

Proof of Theorems 3 and 4. Let us denote $\mathbf{B}=L^{2}\left(\mathbb{R}_{+}, t d t\right)$ the set of measurable functions $a: \mathbb{R}_{+} \rightarrow C$ with norm $|a|_{\mathbf{B}}=$ $\left(\int_{\mathbb{R}_{+}}|a(y, t)|^{2} t d t\right)^{1 / 2}<\infty, \mathscr{M}\left(\mathbb{R}^{n}\right)$ the set of measurable functions defined on $\mathbb{R}^{n}$ valued in $\mathbb{C}$, and $\mathscr{M}\left(\mathbb{R}^{n}, \mathbf{B}\right)$ the set of Bochner-measurable functions $h: \mathbb{R}^{n} \rightarrow \mathbf{B}$. The space $L^{p}\left(\mathbb{R}^{n}, \mathbf{B}\right)(\omega)$ is the set of $h \in \mathscr{M}\left(\mathbb{R}^{n}, \mathbf{B}\right)$ with finite norm

$$
\|h\|_{L^{p}\left(\mathbb{R}^{n}, \mathbf{B}\right)(\omega)}=\left(\int_{\mathbb{R}^{n}}|h(x)|_{\mathbf{B}}^{p} \omega(x) d x\right)^{1 / p} .
$$

We simply name the space as $L^{p}\left(\mathbb{R}^{n}, \mathbf{B}\right)$ when $\omega=1$.

Thus, we can redefine the $g$ as follows:

$$
\begin{gathered}
g(f)(x)=\left\{g_{t} f(x):=Q_{t} f(x)\right\}_{t \in \mathbb{R}_{+}}, \\
Q_{t} f(x):=e^{-t L}(f)(x),
\end{gathered}
$$

which has associated kernel

$$
K(x, y)=\left\{Q_{t}(x, y)\right\}_{t \in \mathbb{R}_{+}} .
$$

It is easy to see that

$$
|K(x, y)|_{\mathbf{B}} \leq \frac{C_{k}}{(1+(|x-y| / \rho(x)))^{k}} \frac{1}{|x-y|^{n}}
$$

for any $k \in N$.

Thus, we can adapt the same argument of Theorems 1 and 2 to prove Theorems 3 and 4; we omit the details here.

\section{Proof of Theorems 5 and 6}

We first need the following lemma.

Lemma 15 (see [5]). Let $k_{t}(x, y)$ be as in (17). For any $N \in \mathbb{N}$, there exists a $C_{N}$ such that

$$
\begin{aligned}
\int_{0}^{\infty} t^{-(n-\beta) / 2-1} k_{t}(x, y) d t \\
\quad \leq \frac{C_{N}}{\left(1+\left(\left|x_{0}-y\right| / \rho\left(x_{0}\right)\right)\right)^{N}} \frac{1}{\left|x_{0}-y\right|^{n-\beta}}
\end{aligned}
$$

for all $x \in B\left(x_{0}, r\right)$ and $y \in \mathbb{R}^{n} \backslash B\left(x_{0}, 2 r\right)$.

Proof of Theorem 5. Without loss of generality, we may assume that $\alpha<0$ and $\omega^{q} \in A_{1+q / p^{\prime}}^{\rho, \theta}(p>1)$. Pick any ball $B=B\left(x_{0}, r\right)$, as the proof of Theorem 1 , we write

$$
f(x)=f_{0}(x)+\sum_{i=1}^{\infty} f_{i}(x)
$$

Hence, we have

$$
\begin{aligned}
& \left(\int_{B\left(x_{0}, r\right)}\left|\mathscr{I}_{\beta} f(x) \omega(x)\right|^{q} d x\right)^{1 / q} \\
& \leq\left(\int_{B\left(x_{0}, r\right)}\left|\mathscr{I}_{\beta} f_{0}(x) \omega(x)\right|^{q} d x\right)^{1 / q} \\
& \quad+\sum_{i=1}^{\infty}\left(\int_{B\left(x_{0}, r\right)}\left|\mathscr{I}_{\beta} f_{i}(x) \omega(x)\right|^{q} d x\right)^{1 / q} .
\end{aligned}
$$

Let $v=q / p$. By the $L_{\omega^{p}}^{p} \rightarrow L_{\omega^{q}}^{q}$ boundedness of $\mathscr{I}_{\beta}$ (see $[10])$, we get

$$
\begin{aligned}
\int_{B\left(x_{0}, r\right)} & \left|\mathscr{I}_{\beta} f_{0}(x) \omega(x)\right|^{q} d x \\
& \leq C\left(1+\frac{r}{\rho\left(x_{0}\right)}\right)^{\alpha} \omega(2 B)^{\nu \lambda}\|f\|_{L_{\alpha, V, \omega}^{p, \lambda}}^{q}\left(\mathbb{R}^{n}\right)
\end{aligned}
$$


From (61), by Corollary 10, then there exists a constant $q_{0}>1$ such that

$$
\begin{aligned}
& \int_{B\left(x_{0}, r\right)}\left|\mathscr{I}_{\beta} f_{i}(x) \omega(x)\right|^{q} d x \\
& \leq C \int_{B\left(x_{0}, r\right)}\left(\int_{0}^{\infty} \int_{B\left(x_{0}, 2^{i+1} r\right) \backslash B\left(x_{0}, 2^{i} r\right)} \mid k_{t}(x, y)\right. \\
& \left.\times f(y) \mid d y t^{\beta / 2-1} d t\right)^{q} \\
& \times \omega(x)^{q} d x \\
& \leq C_{N} \int_{B\left(x_{0}, r\right)} \frac{\left(2^{i} r\right)^{-n}}{\left(1+\left(2^{i} r / \rho\left(x_{0}\right)\right)\right)^{N q}}\left(\int_{B\left(x_{0} 2^{i+2} r\right)}|f(y)|^{p} d y\right)^{q / p} \\
& \times \omega(x)^{q} d x \\
& \leq C_{N}\left(\frac{\omega\left(B\left(x_{0}, 2 r\right)\right)}{\omega\left(B\left(x_{0}, 2^{i+1} r\right)\right)}\right)^{1-\lambda v} \omega\left(B\left(x_{0}, 2 r\right)\right)^{-\lambda v} \\
& \times \frac{\left(1+\left(2^{i} r / \rho\left(x_{0}\right)\right)\right)^{-\alpha+\left(l_{0}+n+4\right) q \theta}}{\left(1+\left(2^{i} r / \rho\left(x_{0}\right)\right)\right)^{N q}}\|f\|_{L_{\alpha, V, \omega}^{p, \lambda}\left(\mathbb{R}^{n}\right)}^{q} \\
& \leq C_{N} 2^{i n(\lambda \nu-1) / q_{0}} \omega\left(B\left(x_{0}, 2 r\right)\right)^{-\lambda v}\|f\|_{L_{\alpha, V, \omega^{p}}^{p, \lambda}}^{q}\left(\mathbb{R}^{n}\right)^{\prime}
\end{aligned}
$$

where $N=2\left(\left[-\alpha+\left(l_{0}+n+4\right) q \theta\right]+1\right)$.

Note that $v \lambda<1$. So

$$
\left\|\mathscr{J}_{\beta} f\right\|_{L_{\alpha, V, \omega q}^{q, \lambda}\left(\mathbb{R}^{n}\right)} \leq C\|f\|_{L_{\alpha, V, \omega}^{p, \lambda}\left(\mathbb{R}^{n}\right)^{.}} .
$$

As for the case $p=1$, the proof is similar.

Proof of Theorem 6. Without loss of generality, we may assume that $\alpha<0$ and $b \in \operatorname{BMO}_{\theta}(\rho)$. Pick any ball $B=$ $B\left(x_{0}, r\right)$, as in the proof of Theorem 1 , we write

$$
f(x)=f_{0}(x)+\sum_{i=1}^{\infty} f_{i}(x)
$$

For $p>1$. Set $\omega \in A_{p}^{\infty, \theta}$. Let $v=q / p$. By the $L_{\omega^{p}}^{p} \rightarrow L_{\omega^{q}}^{q}$ boundedness of $\left[b, I_{\beta}\right]$ (see $[10]$ ), we get

$$
\begin{aligned}
\int_{B\left(x_{0}, r\right)} & \left|\left[b, \mathscr{J}_{\beta}\right] f_{0}(x) \omega(x)\right|^{q} d x \\
& \left.\leq C\left(1+\frac{r}{\rho\left(x_{0}\right)}\right)^{-\alpha} \omega\left(B\left(x_{0}, 2 r\right)\right)^{\nu \lambda}\|f\|_{L_{\alpha, \gamma, \omega^{p}}^{p, \lambda}}^{q} \mathbb{R}^{n}\right)^{.}
\end{aligned}
$$

Set

$$
b_{r}=\frac{1}{\left|B\left(x_{0}, r\right)\right|} \int_{B\left(x_{0}, r\right)} b(x) d x
$$

When $i \geq 1$, by (61) and Corollary 10, then there exists some positive constant $q_{0}>1$ such that

$$
\begin{aligned}
& \int_{B\left(x_{0}, r\right)}\left|\left[b, \mathscr{I}_{\beta}\right] f_{i}(x) \omega(x)\right|^{q} d x \\
& \leq \frac{C_{N}}{\left(1+\left(2^{i} r / \rho\left(x_{0}\right)\right)\right)^{N}}\left(2^{i} r\right)^{-(n-\beta)} \\
& \times\left\{\left(\int_{B\left(x_{0}, r\right)}\left|b(x)-b_{r}\right|^{q} \omega^{q}(x) d x\right) \int_{B\left(x_{0}, 2^{i+1} r\right)}|f(y)| d y\right. \\
& \left.+\omega\left(B\left(x_{0}, r\right)\right) \int_{B\left(x_{0}, 2^{i+1} r\right)}\left|b(y)-b_{r}\right||f(y)| d y\right\} \\
& \leq C_{N} i\|b\|_{\mathrm{BMO}_{\rho}}\left(\frac{\omega\left(B\left(x_{0}, 2 r\right)\right)}{\omega\left(B\left(x_{0}, 2^{i+1} r\right)\right)}\right)^{1-\lambda v} \omega\left(B\left(x_{0}, 2 r\right)\right)^{-\lambda v} \\
& \left.\times \frac{\left(1+\left(2^{i} r / \rho\left(x_{0}\right)\right)\right)^{-\alpha+\left(l_{0}+n+4\right) q \theta}}{\left(1+\left(2^{i} r / \rho\left(x_{0}\right)\right)\right)^{N q}}\|f\|_{L_{\alpha, V, \omega}^{p, \lambda}}^{q} \mathbb{R}^{n}\right) \\
& \leq C_{N} 2^{i n(\lambda \nu-1) / q_{0}} \omega\left(B\left(x_{0}, 2 r\right)\right)^{-\lambda v}\|f\|_{L_{\alpha, V, \omega}^{p, \lambda}}^{q,}\left(\mathbb{R}^{n}\right)^{\prime},
\end{aligned}
$$

by taking $N=2\left(\left[-\alpha+\left(l_{0}+n+4\right) q \theta\right]+1\right)$.

Then,

$$
\left\|\left[b, \mathcal{I}_{\beta}\right] f\right\|_{L_{\alpha, V, \omega}^{q, \lambda}\left(\mathbb{R}^{n}\right)} \leq C\|f\|_{L_{\alpha, V, \omega P}^{p, \lambda}\left(\mathbb{R}^{n}\right)} .
$$

It remains to consider the case $p=1$ and $q=n /(n-\beta)$. Set $\omega \in A_{1}^{\rho, \theta}$. From [10], we know

$$
\begin{aligned}
\omega(\{y & \left.\left.\in \mathbb{R}^{n}:\left|\left[b, \mathscr{I}_{\beta}\right] f(y)\right|>t\right\}\right) \\
& \leq C \Phi\left(\int_{\mathbb{R}^{n}} \frac{|f(x)|}{t} \ln \left(2+\frac{|f(x)|}{t}\right) \Theta(\omega(x)) d x\right),
\end{aligned}
$$

where $\Phi(t)=\left[t \log \left(2+t^{\beta / n}\right)\right]^{n /(n-\beta)}$ and $\Theta(t)=t^{1-\beta / n} \log (e+$ $\left.t^{-\beta / n}\right)$.

From this, we have

$$
\begin{aligned}
& \omega\left(B\left(x_{0}, 2 r\right)\right)^{-\lambda}\left(1+\frac{r}{\rho\left(x_{0}\right)}\right)^{\alpha} \\
& \quad \times \omega\left(\left\{y \in B\left(x_{0}, r\right):\left|\left[b, \mathscr{J}_{\beta}\right] f_{0}(y)\right|>t\right\}\right) \\
& \leq C \sup _{B\left(x_{0}, r\right) \subset \mathbb{R}^{n}}\left(1+\frac{r}{\rho\left(x_{0}\right)}\right)^{\alpha} \omega\left(B\left(x_{0}, 2 r\right)\right)^{-\lambda} \\
& \quad \times \Phi\left(\int_{B\left(x_{0}, 2 r\right)} \frac{|f(x)|}{t} \ln \left(2+\frac{|f(x)|}{t}\right) \Theta(\omega(x)) d x\right) .
\end{aligned}
$$

Set

$$
b_{2^{i+1} r}=\frac{1}{\left|B\left(x_{0}, 2^{i+1} r\right)\right|} \int_{B\left(x_{0}, 2^{i+1} r\right)} b(x) d x
$$


When $i \geq 1$, by (61) and Corollary 10 and Proposition 12, note that $\omega \in A_{1}^{\rho, \theta}$, then there exist some positive constants $q_{0}>1$ and $\eta$ such that

$$
\begin{aligned}
& \omega\left(B\left(x_{0}, 2 r\right)\right)^{-\lambda} t^{-q}\left(1+\frac{r}{\rho\left(x_{0}\right)}\right)^{\alpha} \\
& \times \int_{B\left(x_{0}, r\right)}\left|\left[b, \mathscr{I}_{\beta}\right] f_{i}(x)\right|^{q} \omega(x) d x \\
& \leq \frac{C}{\left(1+\left(2^{i} r / \rho\left(x_{0}\right)\right)\right)^{N q}}\left(2^{i} r\right)^{-n} \omega\left(B\left(x_{0}, 2 r\right)\right)^{-\lambda} \\
& \times\left(1+\frac{r}{\rho\left(x_{0}\right)}\right)^{\alpha} t^{-q} \\
& \times\left\{\int_{B\left(x_{0}, r\right)}\left|b(x)-b_{2^{i+1} r}\right|^{q} \omega(x) d x\right. \\
& \times\left(\int_{B\left(x_{0}, 2^{i+1} r\right)}|f(y)| d y\right)^{q} \\
& +\omega\left(B\left(x_{0}, r\right)\right)\left(\int_{B\left(x_{0}, 2^{i+1} r\right)}\left|b(y)-b_{2^{i+1} r}\right|\right. \\
& \left.\times|f(y)| d y)^{q}\right\} \\
& \leq C\left(1+\frac{2^{i} r}{\rho\left(x_{0}\right)}\right)^{-3 N_{0}}\left(2^{i} r\right)^{-n} \omega\left(B\left(x_{0}, 2 r\right)\right)^{1-\lambda} t^{-q} \\
& \times\left(\left|B_{i}\right|\left\|\frac{\left(b-b_{2^{i+1} r}\right)}{\Phi_{\theta^{\prime}}}\right\|_{\exp L, B_{i}}\|f\|_{L \log L, B_{i}}\right. \\
& \left.+i \int_{B_{i}}|f(y)| d y\right)^{q} \\
& \leq C i^{q}\left(1+\frac{2^{i} r}{\rho\left(x_{0}\right)}\right)^{-3 N_{0}}\left|B_{i}\right|^{-1} \omega\left(B\left(x_{0}, 2 r\right)\right)^{1-\lambda} \\
& \times\left(| B _ { i } | t ^ { - 1 } \operatorname { i n f } _ { \gamma > 0 } \left\{\gamma+\frac{\gamma}{\left|B_{i}\right|} \int_{B_{i}} \frac{|f(y)|}{\gamma}\right.\right. \\
& \left.\left.\times \log \left(2+\frac{|f(y)|}{\gamma}\right) d y\right\}\right)^{q} \\
& +C i^{q}\left(1+\frac{2^{i} r}{\rho\left(x_{0}\right)}\right)^{-3 N_{0}}\left|B_{i}\right|^{-1} \omega(2 B)^{1-\lambda}\left(\int_{B_{i}} \frac{|f(y)|}{t} d y\right)^{q} \\
& \leq C i^{q}\left(\frac{\omega(2 B)}{\omega\left(B_{i}\right)}\right)^{1-\lambda} \omega\left(B_{i}\right)^{-\lambda}\left(1+\frac{r}{\rho\left(x_{0}\right)}\right)^{-2 N_{0}} \\
& \times\left(\int_{B_{i}} \frac{|f(y)|}{t} \log \left(2+\frac{|f(y)|}{t}\right) \omega(y)^{1 / q} d y\right)^{q} \\
& +C i^{q}\left(\frac{\omega(2 B)}{\omega\left(B_{i}\right)}\right)^{1-\lambda} \omega\left(B_{i}\right)^{-\lambda}\left(1+\frac{r}{\rho\left(x_{0}\right)}\right)^{-2 N_{0}} \\
& \times\left(\int_{B_{i}} \frac{|f(y)|}{t} \omega(y)^{1 / q} d y\right)^{q}
\end{aligned}
$$

$$
\begin{gathered}
\leq C i^{q} 2^{-i n(1-\lambda) / q_{0}} \omega\left(B_{i}\right)^{-\lambda}\left(1+\frac{r}{\rho\left(x_{0}\right)}\right)^{\alpha} \\
\times\left(\int_{B_{i}} \frac{|f(y)|}{t} \log \left(2+\frac{|f(y)|}{t}\right)\right. \\
\left.\times \omega(y)^{1 / q} d y\right)
\end{gathered}
$$

by taking $N_{0}=\left[-\alpha+\left(l_{0}+n+4\right) q \theta+\eta\right]+1$ and $N=4 N_{0}$. Hence,

$$
\begin{aligned}
& \omega(2 B)^{-\lambda}\left(1+\frac{r}{\rho\left(x_{0}\right)}\right)^{\alpha} \times \omega\left(\left\{y \in B\left(x_{0}, r\right):\left|\left[b, \mathcal{I}_{\beta}\right]\left(\sum_{i=1}^{\infty} f_{i}\right)(y)\right|>t\right\}\right) \\
& \leq C\left(\sum _ { i = 1 } ^ { \infty } \left(\omega(2 B)^{-\lambda} t^{-q}\left(1+\frac{r}{\rho\left(x_{0}\right)}\right)^{\alpha}\right.\right. \\
&\left.\left.\times \int_{B\left(x_{0}, r\right)}\left|\left[b, \mathscr{I}_{\beta}\right] f_{i}(y) \omega(y)\right|^{q} d y\right)^{1 / q}\right)^{q}\left(\sum _ { i = 1 } ^ { \infty } \left(i^{q} 2^{-i n(1-\lambda) / q_{0}} \omega\left(B_{i}\right)^{-\lambda}\left(1+\frac{r}{\rho\left(x_{0}\right)}\right)^{\alpha}\right.\right. \\
& \times\left(\int_{B_{i}} \frac{|f(y)|}{t} \log \left(2+\frac{|f(y)|}{t}\right)^{q}\right. \\
& \times C \sup _{B\left(x_{0}, r\right) \subset \mathbb{R}^{n}}\left(1+\frac{r}{\rho\left(x_{0}\right)}\right)^{\alpha} \omega(2 B)^{-\lambda} \\
& \times\left(\int_{B\left(x_{0}, r\right)} \frac{|f(x)|}{t} \ln \left(2+\frac{|f(y)|}{t}\right)^{q / q}\right. \\
&\left.\times \omega(y)^{1 / q} d y\right)^{q} \cdot
\end{aligned}
$$

By (73) and (76), we obtain the desired result. This completes the proof.

\section{The Calderón-Zygmund Inequality}

For the open set $\Omega \subset \mathbb{R}^{n}, \omega \in A_{p}^{\rho, \infty}\left(\mathbb{R}^{n}\right)(1 \leq p<f z)$ and $V \in B_{n}$, we say $f \in L_{\alpha, V, \omega}^{p, \lambda}(\Omega)$ if

$$
\begin{aligned}
\|f\|_{L_{\alpha, V}^{p, \lambda}(\Omega)}^{p}= & \sup _{B\left(x_{0}, r\right) \subset \mathbb{R}^{n}}\left(1+\frac{r}{\rho\left(x_{0}\right)}\right)^{\alpha} r^{-\lambda} \\
& \times \int_{B\left(x_{0}, r\right) \cap \Omega}|f(x)|^{p} d x<\infty .
\end{aligned}
$$


In this section, we consider the behavior of the solution of the following Schrödinger equation:

$$
(-\Delta+V) u=f(x), \quad \text { a.e. } x \in \Omega,
$$

where $f \in L_{\alpha, V, \omega}^{p, \lambda, \omega}(\Omega), 1<p<\infty, 0<\lambda<1, \alpha \in(-\infty, \infty)$, and $\omega \in A_{\infty}^{\rho, \infty}$.

Theorem 16. Let $\Omega$ be an open set in $\mathbb{R}^{n}$ and $\alpha \in(-\infty, \infty)$. If $f \in L_{\alpha, V, \omega}^{p, \lambda}(\Omega)$, then there exists a function $u \in L_{\alpha, V, \omega}^{q, v \nu}(\Omega)$, where $1<p<n / 2,1 / p-1 / q=2 / n, v=q / p, 0<\lambda<1 / \nu$, and $\omega^{q} \in A_{1+q / p^{\prime}}^{\rho, \infty}$ such that

$$
(-\Delta+V) u=f(x), \quad \text { a.e. } x \in \Omega .
$$

Furthermore,

$$
\left\|D^{2} u\right\|_{L_{\alpha, V, \omega}^{p, \lambda}(\Omega)} \leq C\|f\|_{L_{\alpha, V, \omega}^{p, \lambda}(\Omega)}
$$

where $1<p<\infty, 0<\lambda<n$, and $\omega \in A_{p}^{\rho, \infty}$;

$$
\|D u\|_{L_{\alpha, V, \omega}^{q, v_{1} \lambda}(\Omega)} \leq C\|f\|_{L_{\alpha, V, \omega}^{p, \lambda}(\Omega)},
$$

where $1<p<n, 1 / p-1 / q=1 / n, v_{1}=q / p, 0<\lambda<n / \nu_{1}$, and $\omega^{q} \in A_{1+q / p}^{\rho, \infty}$;

$$
\|u\|_{L_{\alpha, V, \omega}^{q, v \lambda}(\Omega)} \leq C\|f\|_{L_{\alpha, V, \omega}^{p, \lambda}(\Omega)},
$$

where $1<p<n / 2,1 / p-1 / q=2 / n, v=q / p, 0<\lambda<1 / \nu$, and $\omega^{q} \in A_{1+q / p^{\prime}}^{\rho, \infty}$.

Proof. From the proof of Theorem 5, we have

$$
\|u\|_{L_{\alpha, V, \omega q}^{q, v \lambda}(\Omega)} \leq C\left\|\mathscr{I}_{2} f\right\|_{L_{\alpha, V, \omega}^{q, v \lambda}(\Omega)} \leq C\|f\|_{L_{\alpha, V, \omega}^{p, \lambda}(\Omega)} .
$$

From the proof of Theorem 1, we obtain

$$
\left\|D^{2} u\right\|_{L_{\alpha, V, \omega}^{p, \lambda}(\Omega)} \leq C\left\|D^{2} \mathscr{L}^{-1} f\right\|_{L_{\alpha, V, \omega}^{p, \lambda}(\Omega)} \leq C\|f\|_{L_{\alpha, V, \omega}^{p, \lambda}(\Omega)} .
$$

Thus, (80) and (82) hold.

From page 543 in [1], we know

$$
\left|D_{x} \Gamma(x, y)\right| \leq \frac{C_{k}}{(1+(|x-y| / \rho(x)))^{k}} \frac{1}{|x-y|^{n-1}},
$$

where $\Gamma(x, y)$ is the fundamental solution for $\mathscr{L}=-\Delta+V$.

Using (85) and adapting the argument for Theorem 5 , we then have

$$
\|D u\|_{L_{\alpha, V, \omega}^{q, v_{1} \lambda}(\Omega)} \leq C\left\|D \mathscr{L}^{-1} f\right\|_{L_{\alpha, V, \omega}^{q, v_{1} \lambda}(\Omega)} \leq C\|f\|_{L_{\alpha, V, \omega}^{p, \lambda}(\Omega)} .
$$

Thus, (81) holds. Hence, Theorem 16 is proved.

\section{Conflict of Interests}

The authors declare that there is no conflict of interests regarding the publication of this paper.

\section{References}

[1] Z. W. Shen, " $L^{p}$ estimates for Schrödinger operators with certain potentials," Annales de l'institut Fourier, vol. 45, no. 2, pp. 513546, 1995.

[2] C. Pérez, "Endpoint estimates for commutators of singular integral operators," Journal of Functional Analysis, vol. 128, no. 1, pp. 163-185, 1995.

[3] Y. Komori and S. Shirai, "Weighted Morrey spaces and a singular integral operator," Mathematische Nachrichten, vol. 282, no. 2, pp. 219-231, 2009.

[4] H. Wang, "Intrinsic square functions on the weighted Morrey spaces," Journal of Mathematical Analysis and Applications, vol. 396, no. 1, pp. 302-314, 2012.

[5] L. Tang and J. Dong, "Boundedness for some Schrödinger type operators on Morrey spaces related to certain nonnegative potentials," Journal of Mathematical Analysis and Applications, vol. 355, no. 1, pp. 101-109, 2009.

[6] J. Zhong, Harmonic analysis for some Schrödinger type operators [Ph.D. thesis], Princeton University, 1993.

[7] E. M. Stein, Harmonic Analysis: Real-Variable Methods, Orthogonality, and Oscillatory Integrals, vol. 43, Princeton University Press, Princeton, NJ, USA, 1993.

[8] B. Bongioanni, E. Harboure, and O. Salinas, "Commutators of Riesz transforms related to Schrödinger operators," The Journal of Fourier Analysis and Applications, vol. 17, no. 1, pp. 115-134, 2011.

[9] B. Bongioanni, E. Harboure, and O. Salinas, "Classes of weights related to Schrödinger operators," Journal of Mathematical Analysis and Applications, vol. 373, no. 2, pp. 563-579, 2011.

[10] L. Tang, "Weighted norm inequalities for Schrödinger type operators," Forum Math, 2013.

[11] L. Tang, "Weighted norm inequalities forcommutators of Littlewood-Paley functions related to Schrödinger operators," http://arxiv.org/abs/1109.0100 .

[12] B. Bongioanni, E. Harboure, and O. Salinas, "Weighted inequalities for commutators of Schrödinger-Riesz transforms," Journal of Mathematical Analysis and Applications, vol. 392, no. 1, pp. 622, 2012.

[13] B. Bongioanni, A. Cabral, and E. Harboure, "Lerner's inequality associated to a critical radius function and applications," Journal of Mathematical Analysis and Applications, vol. 407, no. 1, pp. 35-55, 2013. 


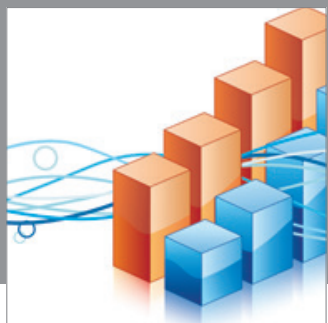

Advances in

Operations Research

mansans

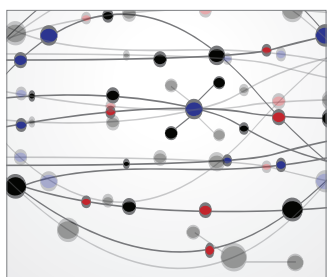

The Scientific World Journal
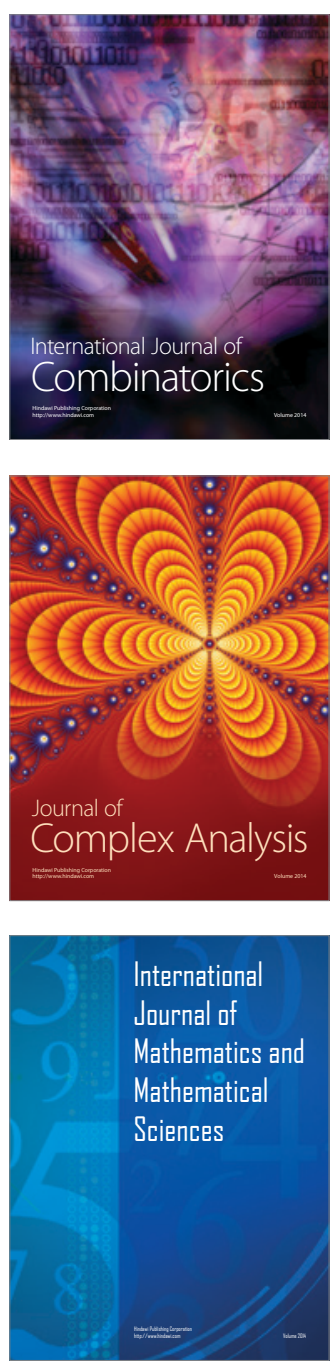
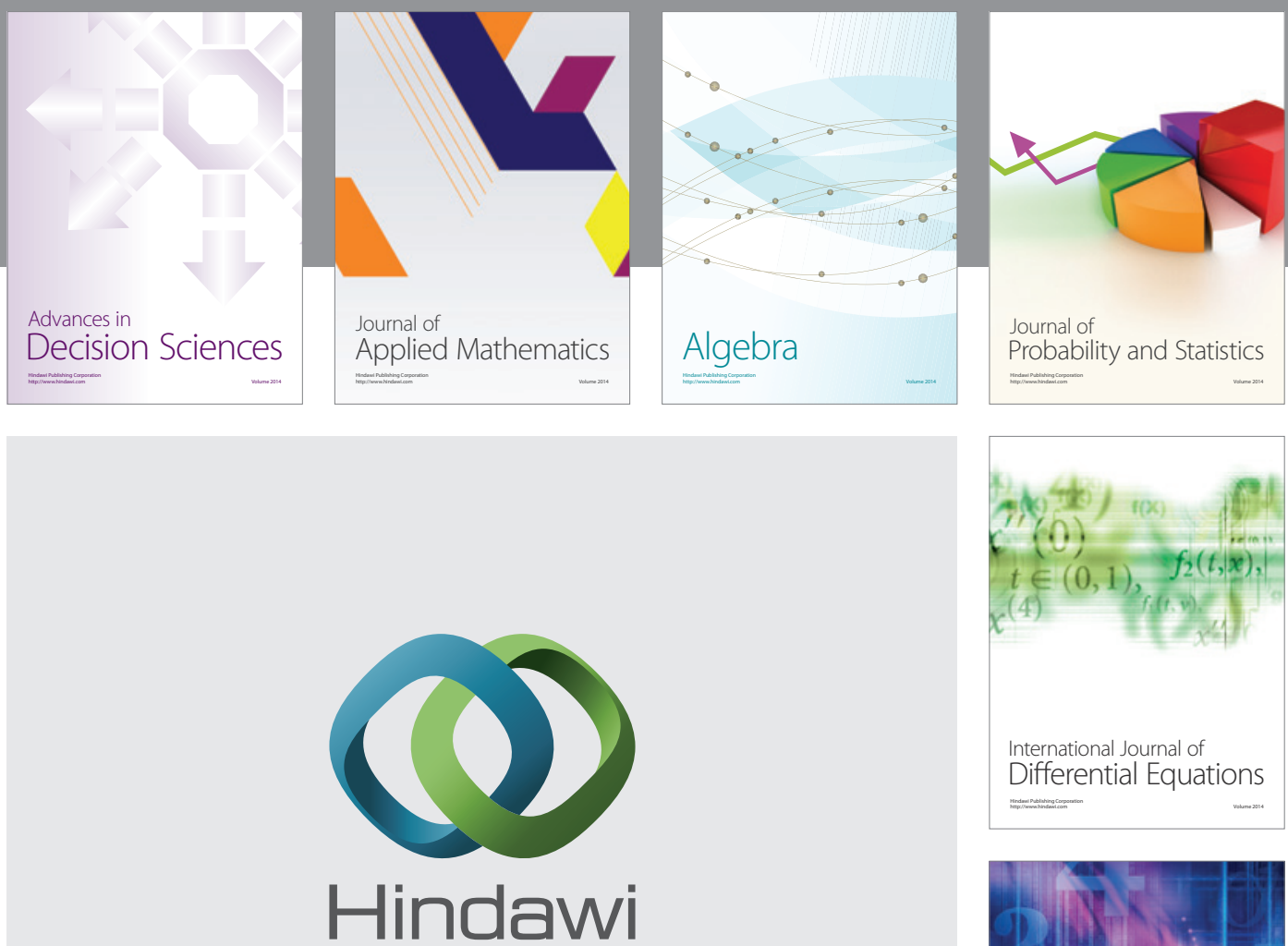

Submit your manuscripts at http://www.hindawi.com
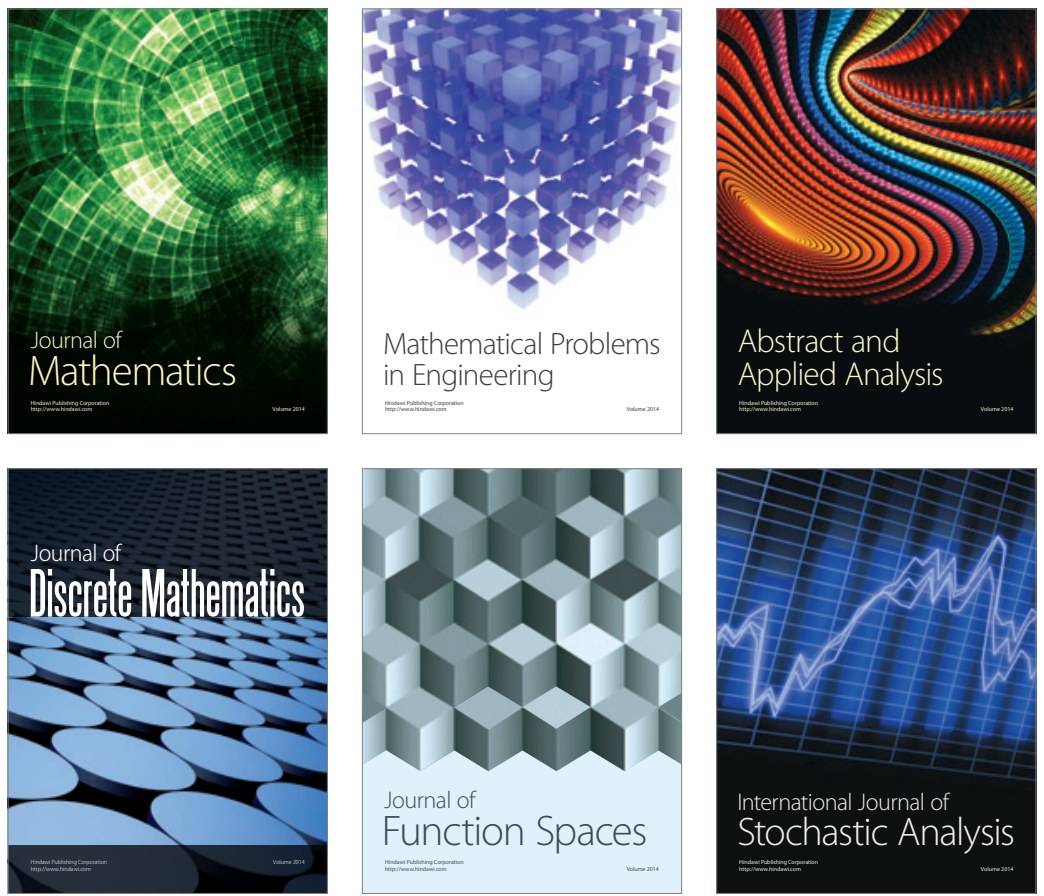

Journal of

Function Spaces

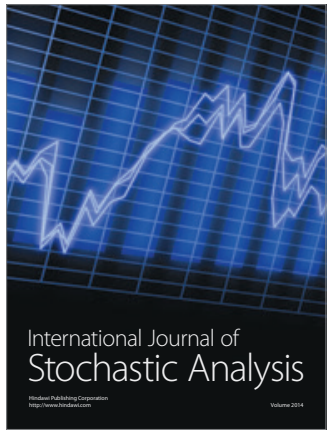

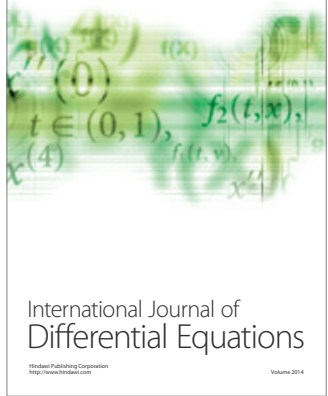
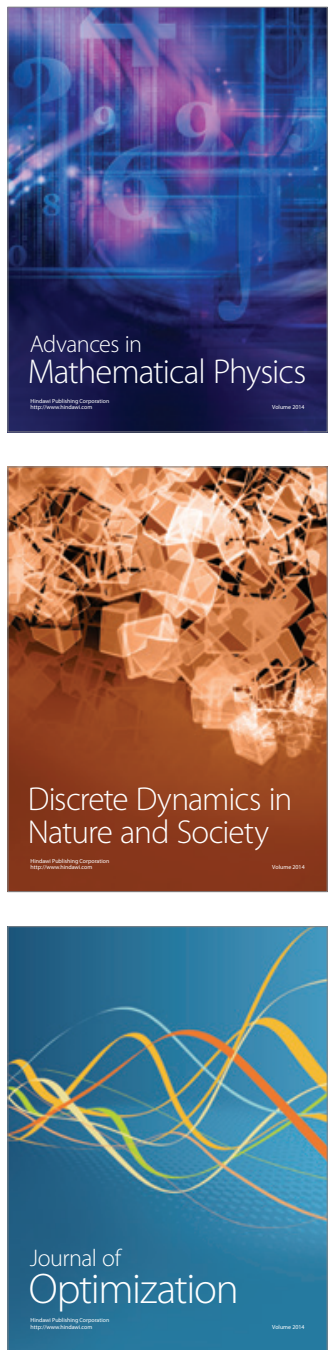